\title{
Hidden varicella outbreak, Luang Prabang Province, the Lao People's Democratic Republic, December 2014 to January 2015
}

\author{
Bounthanom Sengkeopraseuth, ${ }^{a}$ Khonesavanh Bounma, ${ }^{b}$ Chansone Siamong, ${ }^{a}$ Siddhartha Datta, ${ }^{b}$ \\ Bouaphanh Khamphaphongphane, ${ }^{a}$ Phengta Vongphachanh, a Dapeng Luo, ${ }^{b}$ Michael O'Reillyc and \\ Cindy H Chiu \\ Correspondence to Cindy H Chiu (email: DrCindyHChiu@gmail.com).
}

Background: In January 2015, the Lao People's Democratic Republic Ministry of Health received a report of 34 cases of fever and rash with one laboratory-confirmed measles case in Houitone village, Pakseng District of Luang Prabang Province. Between 21 and 27 January, we conducted a field investigation to determine the etiology, magnitude and severity of this outbreak.

Methods: We conducted active case findings in Houitone and neighbouring villages and collected information on age, location, date of rash onset, symptoms and measles vaccination status. We collected serum samples from cases with rash onset of less than 28 days and tested for measles and rubella IgM using enzyme-linked immunosorbent assay.

Results: Between 22 December 2014 and 23 January 2015, 190 fever and rash cases were identified in seven villages in Pakseng District with the majority of the cases in Houitone village. The most affected age group was between 1 and 9 years. The majority of the rashes were vesicular. Of the additional 43 serum samples collected, no samples tested positive for measles or rubella IgM. The clinical manifestation and epidemiology of the disease suggested a varicella outbreak.

Conclusion: The rapid response to a single laboratory-confirmed measles case did not identify a measles outbreak but suggested a varicella outbreak. Low measles vaccination coverage led us to recommend a routine catch-up vaccination campaign. We also recommend collecting information of rash types and photos of rashes in future fever and rash outbreaks to better differentiate potential etiologies.

A cute fever and rash outbreaks have a wide range of possible etiologies and can cause significant morbidity and mortality. Differential diagnosis includes measles that can cause fatality rates as high as $10-30 \%$ and relatively benign diseases such as varicella which rarely result in death $(0.001 \%$ in $5-9$ year-olds and $0.02 \%$ in adults). ${ }^{1}$ Determining the etiology of a fever and rash outbreak can be complicated when there are outbreaks of different etiology occurring simultaneously in the community. ${ }^{2}$

Varicella, caused by the varicella zoster virus, is a common childhood disease characterized by fever and vesicular rash. It mostly affects children aged 1-9 years old and has a mild presentation except in neonates, pregnant women and immunocompromised individuals where varicella can be life-threatening. ${ }^{1,3}$ In developed countries such as the United States of America and Germany, the introduction of varicella vaccination has reduced the disease incidence significantly. ${ }^{4,5}$ However, in an unimmunized population such as the Lao People's Democratic Republic, outbreaks of varicella can be expected, especially during the cooler winter season. ${ }^{6}$

In January 2015, the National Center for Laboratory and Epidemiology (NCLE) of the Lao People's Democratic Republic received a report of 34 cases of fever and rash in the remote village of Houitone (population $=937$ ) in Luang Prabang Province. Measles IgM enzyme-linked immunoabsorbent assay (ELISA) testing of 15 serum samples identified

\footnotetext{
National Center for Laboratory and Epidemiology, Ministry of Health, Vientiane Capital, the Lao People's Democratic Republic.

Office of the WHO Representative in the Lao People's Democratic Republic, Vientiane Capital, the Lao People's Democratic Republic.

Emerging Disease Surveillance and Response Unit, Division of Health Security and Emergencies, World Health Organization Regional Office for the Western Pacific, Manila, Philippines 
one $(6.7 \%)$ measles-positive result; however, reports of vesicular rash in the 34 cases suggested another disease, most likely varicella, may also be circulating. Measles is a high-priority disease in the Lao People's Democratic Republic. Two laboratoryconfirmed measles outbreaks occurred a few months before this report resulting in 369 cases and 12 deaths in Huaphanh and Bolikhamxay Provinces. Following these outbreaks, a nationwide supplemental immunization activity (SIA) for measles and rubella (MR) was completed one month before the Houitone outbreak.

We conducted an outbreak investigation to determine the etiology, magnitude and severity of the Houitone outbreak.

\section{METHODS}

A team consisting of staff from the NCLE and World Health Organization (WHO) joined the provincial and district response teams to investigate the outbreak in Houitone village and neighbouring villages in Pakseng District, Luang Prabang Province between 21 and 27 January 2015.

The clinical case definition of this outbreak was any person presenting with fever and rash between 1 December 2014 and 24 January 2015 in Pakseng District. We conducted active case findings through a door-to-door survey in Houitone village; case findings in neighbouring villages were conducted through review of medical log books at the province and district hospitals and phone interviews with the heads of villages. We collected the name, age, residential location, date of rash onset, symptoms, measles routine and campaign vaccination status of the cases for analysis.

To differentiate between measles and varicella etiologies, information on rash types were collected with photographic documentation in 16 selected cases. Reinvestigation of the laboratory-confirmed measles case was also conducted. Cases were classified according to United States Centers for Disease Control and Prevention (US CDC) and WHO standard case definitions for varicella, measles and rubella (Box 1). ${ }^{3,7}$

Field investigators obtained serum samples from cases whose rash onset was less than 28 days - the optimal time frame for specimen collection to ensure test reliability. ${ }^{8}$ The NCLE laboratory tested the sera for
Box 1. Case definitions of suspected measles, rubella and varicella (adapted from US $\mathrm{CDC}^{3}$ and WHO ${ }^{7}$ )

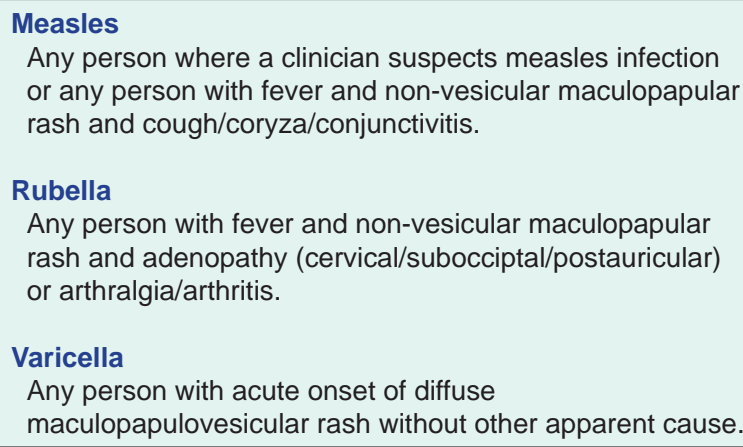

measles and rubella IgM using ELISA (Enzygnost $\AA$ kits, Siemens, Erlangen, Germany).

Houitone village population data were provided by the head of the village for calculation of attack rates (AR). Data cleaning, recoding and descriptive analysis were conducted in Excel (Microsoft Excel, Redmond, USA). Relative risks with 95\% confidence intervals were calculated in Epi-Info 7 (CDC, Atlanta, USA).

\section{RESULTS}

\section{Epidemiological findings}

Between 22 December 2014 and 23 January 2015, 190 cases with fever and rash were identified in seven villages in Pakseng District with the majority of the cases in Houitone village (94.2\%). Response rate of the survey was $94.7 \%$ for those in Houitone village (178/188 households) given 10 absent households. The first two identified cases were an 8-year-old female and a 10-year-old male from the same household in Houitone village with rash onset on 22 December 2014. The case count peaked on 26 December 2014 with 40 cases and a secondary peak appeared on 6 January 2015. The last identified case was a 7-year-old male from Pakseng village with rash onset date of 23 January 2015 (Figure 1).

Ages of the 190 cases ranged from 1.6 months to 30 years (median: 5 years) with the majority between 1 and 9 years ( $n=152,80.0 \%) ; 75$ cases $(39.5 \%)$ were under 5 years with 10 (5.3\%) under 1 year. There were $85(44.7 \%)$ female cases. The overall AR in Pakseng District was $0.9 \%$; the AR in Houitone village was $18.9 \%$. Age-specific AR in this village were $3.7 \%$ 
Figure 1. Epidemic curve of fever and rash outbreak in Pakseng District, Luang Prabang Province, the Lao People's Democratic Republic, December 2014 to January $2015(n=190)$

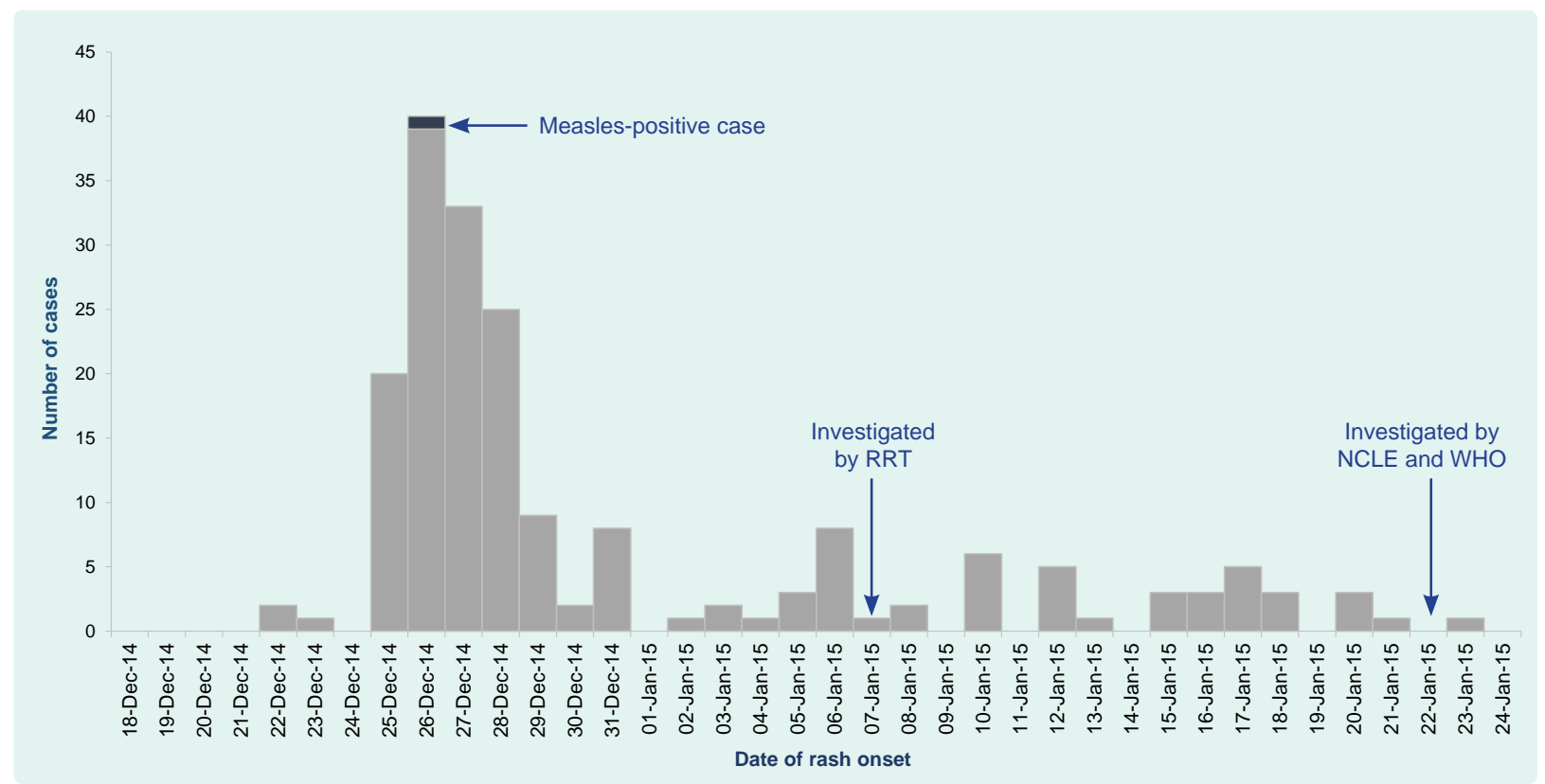

NCLE, National Center for Laboratory and Epidemiology; RRT, Rapid Response Team; and WHO, World Health Organization.

for population who were aged 10 years or older and $69.7 \%$ for the population younger than 10 years (relative risk $=18.8,95 \%$ confidence interval $=12.87-27.54)$.

Reinvestigation of the 5-year-old female case from Houitone village who tested positive for measles IgM revealed that her scars were evidence of vesicular rash characteristic of varicella. She developed a generalized vesicular rash on 26 December 2014 and had a fever soon after. According to her vaccination and MR campaign card, she had been vaccinated for measles through routine immunization on 12 December 2010 and during the SIA on 21 November 2014 (35 days before rash onset and 47 days before sample collection). Five contacts became ill near her symptom onset date ( -1 to 16 days) with similar symptoms of vesicular rash and fever.

\section{Clinical findings}

The majority of the cases presented with vesicular rash ( $n=189,99.5 \%$ ) at either the blistering, scabbing or scarring phase, which is characteristic of varicella (Figure 2), and met the USCDC standard case definition for varicella. ${ }^{3} \mathrm{~A}$ single case manifested with a maculopapular rash $(0.5 \%)$ without cough, coryza or conjunctivitis and met the WHO standard case definition for rubella. ${ }^{7}$ NocasesmettheWHOstandardcasedefinitionformeasles. ${ }^{7}$
The severity of the illness of the cases was uniformly mild with no complications, hospitalization or death.

\section{Laboratory findings}

Of the additional 43 serum samples collected, no (0\%) samples tested positive for measles, and four (9.3\%) were equivocal for measles IgM. No (0\%) samples tested positive for rubella, and six (14.0\%) were equivocal for rubella IgM.

\section{Measles vaccination coverage}

Of the 190 cases, 84 (44.2\%) had evidence of measles vaccination, including 30 ( $15.8 \%$ ) verified by vaccination card and $54(28.4 \%)$ by self-reporting. Among cases who were age-eligible (9 months to 10 years) to receive MR vaccination during the SIA $(n=152,80.0 \%)$, $107(70.4 \%)$ reportedly received the MR vaccination.

\section{DISCUSSION}

Despite the initial laboratory findings that suggested a measles outbreak, the epidemiological and clinical evidence suggested this outbreak was due to varicella. Evidence supporting this includes: (1) the nature of the rash in all but one case was vesicular; (2) the illness was mild and lacked complications; and (3) reinvestigation of 
Figure 2. Cases with different phases of vesicular rash in the fever and rash outbreak in Pakseng District, Luang Prabang Province, the Lao People's Democratic Republic, December 2014 to January 2015

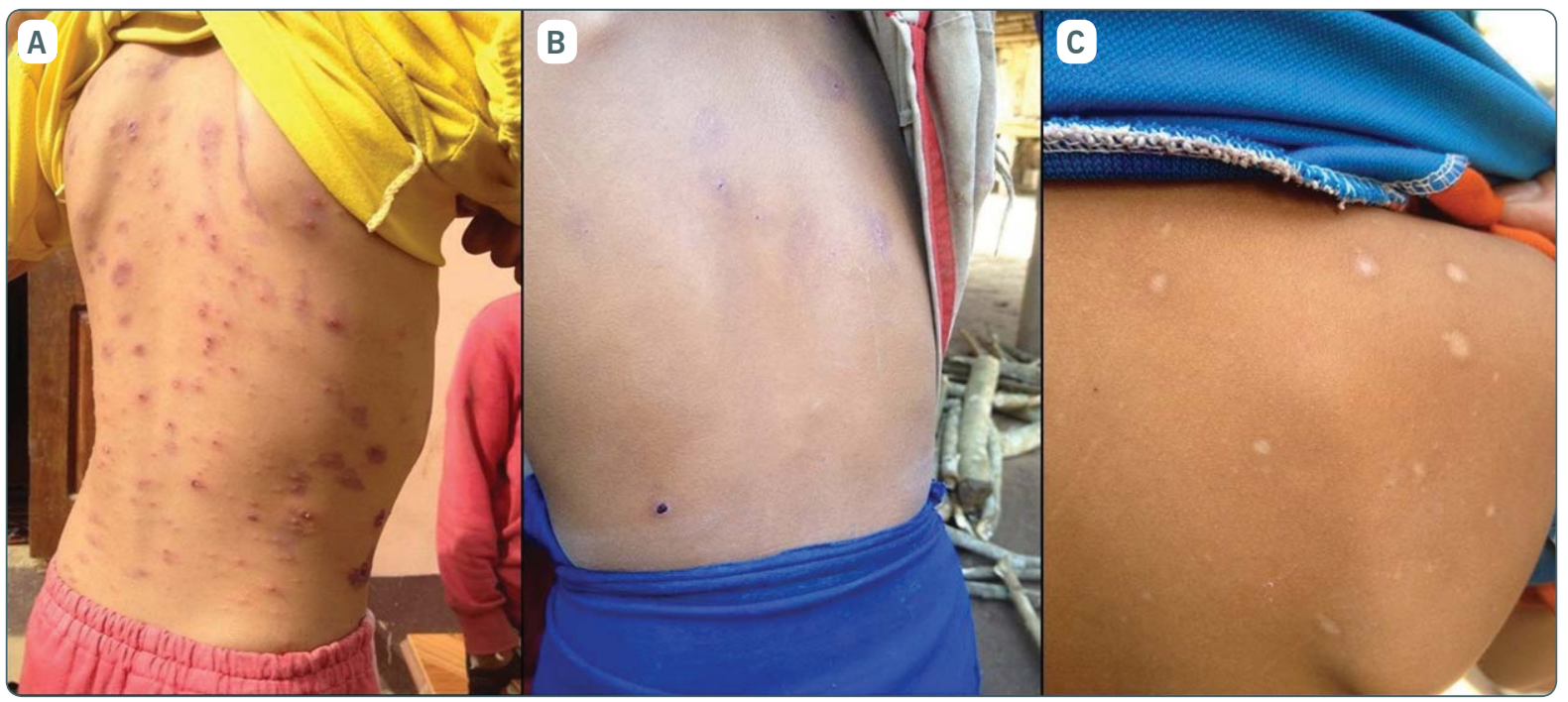

A: blistering phase; B: scabbing phase; and C: scarring phase.

the case with the positive measles IgM result identified inconsistent clinical presentation and epidemiological linkage to other vesicular rash cases. Given her recent MR vaccination, this most likely was a false positive. $^{9}$

Varicella typically presents during the cooler winter season or in regions with temperate climate; the disease predominantly affects pre-adolescent children in temperate climates as opposed to appearing later in life in tropical climates. ${ }^{6,10}$ The wide age range of the cases in this outbreak is consistent with this pattern and similar to other tropical South-East Asia countries such as Malaysia, the Philippines and Thailand where more than $90 \%$ seroconversion can only be seen in those older than 30 years. ${ }^{11-13}$

In a measles elimination setting, according to the WHO Western Pacific Region Measles Elimination Field Guide, a single laboratory-confirmed case requires immediate investigation and response. ${ }^{14}$ The investigation conducted after the laboratory-confirmed measles case is a reflection of the adherence of NCLE to the WHO guidelines. In the Lao People's Democratic Republic, the national measles vaccination coverage was $87 \%$ in 2014 and $88 \%$ for the Pakseng District. The nationwide measles SIA conducted in November 2014 reported vaccination coverage of $100 \%$ nationwide and $105 \%$ in Pakseng District (Correspondence with the Lao People's Democratic Republic Ministry of Health, August 2015). Although the Houitone outbreak was not due to measles, we detected a suboptimal and much lower routine and campaign vaccination coverage, highlighting the importance to strengthen both routine immunization and SIA.

In some developing countries such as the Lao People's Democratic Republic, varicella vaccination has not been incorporated into routine immunization programmes as the cost of vaccine outweighs the public health benefit. However, there is some evidence of higher rates of complications in varicella outbreaks among rural South-East Asian populations with largely naïve populations. ${ }^{15}$ In the following three months after this outbreak, at least 16 additional fever and vesicular rash outbreaks were documented with no reported deaths in the Lao People's Democratic Republic (unpublished data). Continued surveillance and investigation of fever and vesicular rash cases is necessary to monitor for severe health outcomes and reassess the need for varicella vaccination programmes.

There are several limitations in this study. First, the door-to-door survey was conducted only in Houitone village which may underestimate the scope of the outbreak. Second, during the door-to-door survey, we encountered $5.3 \%$ absent households which may underestimate the attack rate for the village. There may also be a certain level of recall bias regarding the rash onset date that could potentially limit the yield in ELISA IgM test if the true rash onset date was more than 28 days before the test. 
In summary, the rapid response to a measlespositive laboratory result discovered a varicella outbreak. The low measles vaccination coverage detected in this setting led us to recommend a routine catchup vaccination campaign. We recommend collecting detailed information of rash type and obtaining photo documentation of lesions to better differentiate potential etiologies of future fever and rash outbreaks.

\section{Conflicts of interest}

None declared.

\section{Funding}

This outbreak investigation was funded by WHO.

\section{Acknowledgements}

We would like to thank the following: $\mathrm{Dr}$ Gregory Jennings and $\mathrm{Dr}$ Manilay Phengxay from WHO for their input on the laboratory and epidemiological aspects of the investigation, respectively; and the Institute Pasteur of the Lao People's Democratic Republic for officially informing the Ministry of Health of the laboratory findings that confirmed the varicella etiology in this outbreak from their independent study, especially Dr Darouny Phonekeo, Ms Phonethipsavanh Nouanthong and Ms Keooudomphone Vilivong who conducted the varicella testing in their study. We would also like to thank the provincial and district health offices and hospitals for sharing their data and joining the investigation. Finally, we would like to thank the village heads and village volunteers who helped with translation and data collection.

\section{References}

1. Heymann DL. Control of communicable diseases manual, 19th Edition. Washington, DC, American Public Health Association, 2008.

2. Gupta SN, Gupta N, Gupta S. Concurrent multiple outbreaks of varicella, rubeola, german measles in unvaccinated children of coeducational mount carmel senior secondary school, Thakurdwara
Palampur of northern Himachal, India. Journal of Family Medicine and Primary Care, 2015, 4:117-123. doi:10.4103/22494863.152267 pmid:25811001

3. Lopez A, Schmid S, Bialek S. Chapter 17: Varicella. In: Vaccine preventable diseases surveillance manual, 5th Edition. Atlanta, United States Centers for Disease Control and Prevention, 2011 (http://www.cdc.gov/vaccines/pubs/surv-manual/chpt17-varicella. pdf, accessed 4 December 2015).

4. Baxter R et al. Impact of vaccination on the epidemiology of varicella: 1995-2009. Pediatrics, 2014, 134:24-30. doi:10.1542/peds.2013-4251 pmid:24913796

5. Streng $A$ et al. Varicella routine vaccination and the effects on varicella epidemiology - results from the Bavarian Varicella Surveillance Project (BaVariPro), 2006-2011. BMC Infectious Diseases, 2013, 13:303. doi:10.1186/1471-2334-13-303 pmid:23815523

6. Lee BW. Review of varicella zoster seroepidemiology in India and Southeast Asia. Tropical Medicine \& International Health, 1998, 3:886-890. doi:10.1046/j.1365-3156.1998.00316.x pmid:9855401

7. WHO-recommended standards for surveillance of selected vaccine-preventable diseases. Geneva, World Health Organization, 2003 (http://apps.who.int/iris/bitstream/10665/68334/1/WHO V-B_03.01_eng.pdf?ua=1, accessed 4 December 2015).

8. Mokhatari-Azad $\mathrm{T}$ et al. Optimal timing of specimen collection after rash onset for diagnosis of measles IgM antibody. Archives of Razi Institute, 2005, 60:1-10.

9. Helfand RF et al. Timing of development of measles-specific immunoglobulin $\mathrm{M}$ and $\mathrm{G}$ after primary measles vaccination. Clinical and Diagnostic Laboratory Immunology, 1999, 6:178180.

10. Lolekha $\mathrm{S}$ et al. Effect of climatic factors and population density on varicella zoster virus epidemiology within a tropical country. The American Journal of Tropical Medicine and Hygiene, 2001, 64:131-136. pmid: 11442207

11. Malik YA, Baharin R. Prevalence of varicella zoster virus infection in Malaysia. Johannesburg, Proceedings of the 5th International Congress on the Impact of Viral Disease in the Developing World, 1995.

12. Barzaga NG, Roxas JR, Florese RH. Varicella zoster virus prevalence in metro Manila, Philippines. Journal of the American Medical Association, 1994, 274:S633-S635.

13. Migasena $\mathrm{S}$ et al. Seroprevalence of varicella zoster virus antibody in Thailand. International Journal of Infectious Diseases, 1997, 2:26-30. doi:10.1016/S1201-9712(97)90007-2

14. Measles elimination field guide. Manila, World Health Organization Regional Office for the Western Pacific, 2013 (http://www.wpro. who.int/immunization/documents/measles elimination field guide_2013.pdf, accessed 4 December 2015).

15. Mandal BK et al. Adult susceptibility to varicella in the tropics is a rural phenomenon due to the lack of previous exposure. The Journal of Infectious Diseases, 1998, 178 Suppl 1;S52S54. doi:10.1086/514262 pmid:9852974 